\title{
LA LECTURA Y LA ESCRITURA: \\ UN ESTUDIO SOBRE REPRESENTACIONES SOCIALES DE ESTUDIANTES UNIVERSITARIOS
}

READING AND WRITING:

A STUDY OF UNIVERSITY STUDENTS SOCIAL REPRESENTATIONS

\author{
Volumen 15, Número 2 \\ Mayo - Agosto \\ pp.1-26
}

Este número se publicó el $1^{\circ}$ de mayo de 2015

DOI: http://dx.doi.org/10.15517/aie.v15i2.18959

Karina Savio

Revista indizada en REDALYC, $\underline{\text { SCIELO }}$

Revista distribuida en las bases de datos:

CATÁLOGO DE LATINDEX, IRESIE, CLASE, DIALNET, DOAJ, E-REVIST@S, SHERPA/ROMEO, QUALIS, MIAR

Revista registrada en los directorios:

ULRICH'S, $\underline{\text { REDIE}}, \underline{\text { RINACE}}, \underline{\text { OEI }}, \underline{\text { MAESTROTECA, PREAL, }}$ 


\title{
LA LECTURA Y LA ESCRITURA: UN ESTUDIO SOBRE REPRESENTACIONES SOCIALES DE ESTUDIANTES UNIVERSITARIOS

\author{
READING AND WRITING:
} \\ A STUDY OF UNIVERSITY STUDENTS SOCIAL REPRESENTATIONS
}

\begin{abstract}
Karina Savio ${ }^{1}$
Resumen: En este artículo, nos proponemos describir y analizar las representaciones sociales de un grupo de estudiantes de la Universidad Nacional Arturo Jauretche, de Argentina, con el objetivo de desentrañar tanto el modo en que estos estudiantes se perciben como lectores y escritores, como el modo en que conciben estas prácticas académicas. Consideramos que estas construcciones imaginarias inciden en el trabajo concreto de los estudiantes y en sus estudios universitarios, por lo que es necesario operar sobre ellas. Para tal fin, hemos elaborado una encuesta con preguntas abiertas y hemos aplicado este instrumento a 303 alumnos ingresantes y a 171 alumnos que concluían su primer año de estudio. El propósito de aplicar un cuestionario en dos momentos temporales diferentes estuvo en función de evaluar continuidades y rupturas sobre los modos de pensar estas prácticas. La lectura y la escritura en la educación superior son dos prácticas que, en las últimas décadas, han sido abordadas por numerosos estudios dentro del ámbito académico. Entre los resultados que hemos obtenido, se ha evidenciado, por un lado, que, al término del año académico, ciertas representaciones y prácticas vinculadas a la lectura y a la escritura todavía persisten y, por el otro, que emergen singularidades características del proceso académico, en el que el alumno, en tanto sujeto universitario en formación, comienza a incorporarse en la comunidad discursiva de este ámbito.

Palabras clave: REPRESENTACIONES SOCIALES, LECTURA, ESCRITURA, EDUCACIÓN SUPERIOR, ARGENTINA

Abstract: In this paper, we intend to describe and analyze the social representations of a group of students from the Universidad Nacional Arturo Jauretche, of Argentina, with the aim to enquire how these students perceive themselves as readers and writers and how they conceive these academic practices. We consider that these imaginary constructions affect the actual work of students and their university studies, so it is necessary to operate on them. To this end, we elaborated a survey with open questions and we have used this instrument in 303 new students and in 171 students that concluded their first year of study. The purpose of applying a questionnaire in two different temporal moments was to evaluate the continuities and ruptures about these practices conceptions. Reading and writing in higher education are two practices that, in recent decades, have been examined by numerous studies within the academia. From the results we have obtained, we observe, on the one hand, that, at the end of the academic year, certain representations and practices related to reading and writing persist and, on the other, that singularities characteristics of the academic process emerge, in which the student, as an academic training subject, begins to join the university discourse community.
\end{abstract}

Key words: SOCIAL REPRESENTATIONS, READING, WRITING, HIGHER EDUCATION, ARGENTINA

\footnotetext{
${ }^{1}$ Investigadora del CONICET en Argentina. Licenciada en Letras y Licenciada en Psicología, Doctora de la Universidad de Buenos Aires de Argentina. Trabaja en la Universidad Nacional Arturo Jauretche y en la Universidad de Buenos Aires, Argentina.
}

Dirección electrónica: karinasavio@fibertel.com.ar

Artículo recibido: 17 de julio, 2014

Enviado a corrección: 4 de diciembre, 2014

Aprobado: 20 de abril, 2015 


\section{Introducción}

Desde hace ya algunas décadas, la preocupación por la lectura y por la escritura en la educación superior se ha convertido en una interrogante reiterada en el mundo académico, la cual se ha plasmado en la elaboración de un sinfín de manuales -tendientes a transformar el discurso académico en objeto de aprendizaje- y en la creación de talleres, cursos y/o materias de lecto-escritura dentro de los planes de estudio de instituciones tanto públicas como privadas ${ }^{2}$. Estos manuales, que transitan los pasillos de la academia, y la aparición de asignaturas y talleres relativos a la discursividad universitaria presuponen, a la vez que construyen, un ideal de estudiante, a saber: lector analítico y escritor avezado en las convenciones académicas. Proyectan, en este mismo gesto, el surgimiento de un sujeto universitario.

Esta relación entre una identidad -la universitaria- y la comunidad discursiva en la que el sujeto se inscribe ha sido analizada por diversos autores (Hyland, 2009; Ivanič, 1998; Swales, 1990). Ivanič $(1998,45)$ señala, en este sentido, que las características de los distintos discursos están moldeadas por los diferentes intereses, valores, creencias y prácticas de los grupos sociales particulares y posicionan, entonces, a los escritores como participando de esos intereses, valores, creencias y prácticas. De esta manera, cuando un escritor utiliza determinados términos y/o estructuras, el escritor se alinea con aquellos que usan los mismos términos y/o estructuras, construye una identidad sobre sí mismo $\mathrm{y}$, por ende, se incluye en una comunidad discursiva singular. En efecto, en el caso de las instituciones superiores, la naturaleza de esta identidad está configurada, entre otras variables, por las prácticas de lectura y escritura que allí circulan.

En este artículo presentamos los resultados de una investigación que persigue como propósito general analizar las representaciones sociales de un grupo de estudiantes de la Universidad Nacional Arturo Jauretche (UNAJ), emplazada en la provincia de Buenos Aires en Argentina, acerca de la lectura y de la escritura. Nos preguntamos por la imagen que el estudiante universitario percibe de sí mismo como lector/escritor y de las prácticas de lectura y escritura que realiza en dos momentos significativos de su trayecto universitario: en su ingreso a la institución y un año después, al finalizar la cursada de un taller obligatorio de lectura y escritura. Consideramos que este imaginario -encarnado en diferentes representaciones- incide de manera positiva y/o negativa en la práctica concreta de los

\footnotetext{
${ }^{2}$ Para un desarrollo de la situación argentina en relación con la enseñanza de la escritura académica, véase Carlino (2006).
} 
alumnos y en sus estudios universitarios, y participa en la configuración de la identidad de los estudiantes y su posicionamiento como miembros de la comunidad académica. Identificar las construcciones imaginarias en torno a estas prácticas permite operar sobre ellas.

Un dato relevante a destacar es que esta universidad abrió sus puertas recientemente, en el año 2011, producto de las políticas educativas de inclusión social que han surgido en la última década en este país. De hecho, el objetivo explícito de esta institución lo constituye la integración, inclusión y promoción social de los sectores populares que viven en el municipio de Florencio Varela. En este contexto, el perfil de los estudiantes que ingresan, en su mayoría, corresponde a la primera generación de estudiantes universitarios/as y/o tericarios/as en la historia de sus familias ${ }^{3}$, por cuanto provienen de los distritos más pobres del Gran Buenos Aires (Ceresani et al., 2013). Por lo general, presentan historias de abandono y reinserciones a lo largo de su escolaridad y, en algunos casos, han finalizado la educación secundaria con logros precarios.

Los estudiantes de esta universidad se relacionan con la lectura y la escritura a través de un curso de ingreso y de un taller. En primera instancia, los ingresantes deben asistir a un Curso de Preparación Universitaria de Lengua que tiene una duración aproximada de un mes y medio, en el que se realiza una breve introducción sobre las nociones de género discursivo y tipos textuales y se revisan temáticas vinculadas con la normativa de la lengua (ortografía, acentuación, puntuación, clases de palabras y sintaxis). Luego, al ingresar a la universidad, cursan un ciclo inicial -obligatorio para todas las carreras- conformado por cuatro materias: Prácticas culturales, Matemática, Problemas de historia argentina y el Taller de Lectura y Escritura.

El objetivo principal de dicho Taller, que tiene una duración cuatrimestral, es que los alumnos del primer año adquieran y desarrollen algunas habilidades indispensables a la hora de comprender y producir textos propios del ámbito académico. Esta materia busca que los estudiantes conozcan y manejen algunas convenciones de estos textos entendidas como claves para su lectura y su escritura. En tal sentido, se abordan diferentes géneros discursivos -la entrada de enciclopedia, el artículo de divulgación, el capítulo de manual, la entrada de diccionario especializado, el ensayo, la nota de opinión, el artículo académico- y los tipos textuales más frecuentes en la universidad: la explicación y la argumentación.

${ }^{3}$ En el 2011 , el $94 \%$ de estudiantes que ingresaron a la UNAJ se caracterizaba por ser primera generación de universitarios/as y terciarios/as en sus familias de orígenes. Esa tendencia se ha ido modificando gradualmente y el porcentaje fue disminuyendo durante $2012(77 \%)$ y $2013(72 \%)$. Sin embargo, los valores siguen siendo altos. (Ceresani et al., 2013) 
Finalmente, después de presentar y trabajar con la complementación y la confrontación de fuentes, se les solicita a los alumnos realizar un informe de lectura sobre alguna problemática específica respetando el formato académico.

Ahora bien, en los siguientes apartados, presentaremos, en primer lugar, el marco teórico y el encuadre metodológico en el que inscribimos esta investigación y las encuestas realizadas. En una segunda instancia, desarrollaremos los resultados de la investigación y los discutiremos siguiendo el eje prácticas académicas de lectura y escritura-sujeto universitario.

\section{Marco teórico}

En Argentina, múltiples trabajos han abordado la compleja relación entre las prácticas de lectura y escritura, y la universidad. Contamos, en efecto, con diversos especialistas que han investigado esta problemática y con carreras de especialización cuyo objetivo principal ha sido la formación de docentes en el área ${ }^{4}$. En cuanto a los trabajos teóricos producidos podríamos señalar, por un lado, un grupo de investigaciones en el que predomina un criterio descriptivo, dado que analiza un corpus previamente delimitado con el fin de desentrañar los rasgos que singularizan los textos producidos por los estudiantes y las estrategias por ellos empleadas y, por el otro, estudios que presentan una finalidad principalmente pedagógica, articulada con propuestas para talleres de escritura.

Los temas que se han abordado han sido, entre otros, el proceso de lectura a partir de apuntes (Arnoux y Alvarado, 1997, 1998 y 1999) y el de escritura a partir de los borradores (Carlino, 2003c), el dispositivo enunciativo en la comprensión y producción de textos (Arnoux, Nogueira y Silvestri, 2002), la reformulación en las síntesis (Arnoux, Nogueira y Silvestri, 2006), la polifonía en la escritura de textos argumentativos y la formulación de preguntas (Arnoux, Nogueira y Silvestri, 2001, 2007), las tradiciones pedagógicas (Arnoux, Nogueira y Silvestri, 2004), los desafíos que la lectura de textos científicos y académicos plantea a los ingresantes (Carlino, 2003a y 2003b).

Por otra parte, la problemática de las representaciones sociales de los estudiantes sobre la lectura y la escritura no ha sido relegada dentro de las investigaciones académicas. Por el contrario, diversos trabajos han intentado rastrear este imaginario en diferentes

\footnotetext{
${ }^{4}$ Es de destacar el trabajo realizado hasta la fecha en la Universidad de Buenos Aires por la Dra Elvira Narvaja de Arnoux y su equipo de investigación. Para una ampliación sobre la historia de la cátedra de Semiología y el Taller de Lectura y Escritura, véase Pereira (2006).
} 
poblaciones universitarias (Bidiña y Zerillo, 2013; Di Stefano y Pereira, 1998; Magnanego y Savio, 2006). Sin embargo, hasta el momento ninguna investigación ha tomado como objeto de estudio las representaciones de los alumnos concurrentes a la Universidad Nacional Arturo Jauretche. Entendemos, en este sentido, que las prácticas de lectura y escritura poseen una doble naturaleza, cognitiva e histórico-social (Arnoux, 2009), por lo que se encuentran atravesadas por numerosas dimensiones: procesos de índole cognitivo, cuestiones socioculturales, variables históricas, que deben ser consideradas en el momento del análisis.

En esta investigación, para determinar el modo en que los estudiantes se conciben como lectores y escritores, y el modo en que piensan las prácticas de lectura y de escritura, partimos, entonces, del concepto de representación social. Al igual que Castorina, Barreiro y Toscano (2005), consideramos que la representación se define como una forma de conocimiento socialmente elaborada, que construye una realidad social y permite comprenderla. Es orientadora de la conducta y de la comunicación entre los sujetos en el mundo. De esta forma, está relacionada con la percepción y constituye la base de las creencias sociales (Raiter, 2002, p.11). Cabe señalar que esta noción proviene de la psicología social (Moscovici, 1989; Jodelet, 1989) y que, con posterioridad, fue empleada y reformulada por otros campos de conocimiento, tales como la sociología y la lingüística ${ }^{5}$.

Ahora bien, para los fines de nuestro análisis, nos interesa destacar cuatro características relevantes que se han estudiado en torno a las representaciones: su carácter mutable, su relación con las prácticas sociales, su lugar en la configuración de la identidad social y su materialidad discursiva.

En primer lugar, las representaciones, definidas como estructuraciones significantes, presentan una particularidad: no son estables. En efecto, son concebidas como construcciones cuyo carácter conflictivo es esencial en su proceso de formación, lo que implica pensar una dinámica en permanente tensión (Moscovici y Marcová, 2003, p.135). Estos cambios -a nivel de las representaciones- pueden ser el resultado de diferentes procesos, entre ellos, la enseñanza en los contextos didácticos.

Las representaciones sociales, en segundo lugar, favorecen la comprensión y la comunicación entre los sujetos, y, a su vez, operan sobre sus comportamientos. Según Bourdieu (1985), son formas de percepción y evaluación, de conocimiento y re-conocimiento

\footnotetext{
${ }^{5}$ Para una ampliación respecto de las representaciones lingüísticas, véase Arnoux y Bein (1999).
} 
socialmente compartidas como saberes prácticos, que actúan sobre la estructuración del mundo. De allí que las representaciones y las prácticas estén en permanente interrelación.

A su vez, estas representaciones, según Castorina, Barreiro y Toscano (2005), otorgan un conjunto de significaciones que delimitan las posiciones de los sujetos en su articulación con los grupos, configurando la identidad social. De esta forma, a través de las representaciones, los miembros de un grupo negocian sus identidades.

Por último, las representaciones sociales son implícitas, es decir, los sujetos no son conscientes de su existencia. Un modo de acceder a su estudio es, entonces, a través del lenguaje. Por ello, el investigador, a partir de diferentes técnicas y herramientas, puede rastrear su emergencia en la materialidad discursiva y construir las representaciones que subyacen en el imaginario de un grupo.

\section{Metodología}

\subsection{El instrumento}

Diversos instrumentos pueden ser empleados a la hora de acceder a las representaciones sociales de un grupo: entrevistas grupales e individuales, dirigidas, semidirigidas; cuestionarios autoadministrados; asociación libre de palabras, entre otros. Para seleccionar y confeccionar la herramienta a implementar en nuestra investigación hemos tomado en cuenta dos cuestiones primordiales: la amplitud de la muestra, por un lado, y nuestras propias presuposiciones sobre las prácticas de lectura y de escritura de los estudiantes, y sobre la imagen que ellos mismos tienen como lectores y escritores, por el otro.

Por esta razón, hemos optado por diseñar un cuestionario breve con preguntas abiertas. En este sentido, los cuestionarios -en nuestro caso, autoadministrados- nos permiten recoger una gran cantidad de datos de un número considerable de sujetos, quienes, por otra parte, responden de manera anónima. A su vez, las preguntas abiertas evitan orientar las respuestas del encuestado y plasmar la visión del encuestador. Las dos únicas representaciones proyectadas en el armado de las encuestas son: 1. La lectura y la escritura pueden presentar alguna clase de dificultad; 2. Se pueden producir cambios en los modos de leer y de escribir (sólo figura en la segunda).

El primer cuestionario se realizó en febrero del 2013, el primer día en que los alumnos ingresantes asistieron al módulo de Lengua del Curso de Preparación Universitaria de la UNAJ. En esta encuesta, los estudiantes debían completar algunos datos personales (edad, 
sexo, escolaridad) y debían especificar si habían asistido a cursos de lectura y/o de escritura anteriores. Luego, se les preguntaba lo siguiente:

1. ¿Le gusta leer? Si es así, ¿qué tipo de lecturas prefiere?

2. ¿Escribe a menudo? ¿En qué momentos?

3. ¿Tiene alguna dificultad con la lectura o la escritura? ¿Cuáles?

4. ¿Qué expectativas tiene en relación con este curso?

La segunda encuesta se aplicó a un grupo de estudiantes cursantes del Taller de Lectura y Escritura al finalizar el primer año de carrera. Por tanto, estos alumnos ya habían adquirido cierta experiencia universitaria y estaban finalizando la cursada de una asignatura dedicada a la adquisición de competencias lecto-escritoras. Se efectuó a fines de noviembre del mismo año. Esta encuesta fue similar a la anterior, se solicitaron algunos datos personales (edad, sexo, escolaridad) y, luego, se incluyeron las mismas preguntas. La única diferencia consistió en una modificación a la pregunta 4:

4. ¿Cuál es su opinión sobre el Taller de Lectura y Escritura? ¿Hubo cambios en su escritura o en la lectura a partir del Taller? ¿Cuáles?

El propósito de emplear el mismo cuestionario a estos dos grupos estuvo en función de evaluar cambios en la materialidad discursiva, con el fin de detectar continuidades y rupturas sobre los modos en los que los estudiantes conciben la lectura y la escritura luego de haber asistido a un curso y a una asignatura vinculados con estas problemáticas y cursado un año de universidad.

\subsection{Tipo de análisis}

La investigación que desarrollamos contempla aspectos cuantitativos como aspectos cualitativos. En un primer momento, con base en lo que los mismos estudiantes respondieron elaboramos una matriz de datos $^{6}$, en la que establecimos categorías que permitiesen la sistematización de la información para su posterior análisis. La construcción de esta matriz fue el resultado de un trabajo exploratorio tendiente a identificar regularidades en las

\footnotetext{
${ }^{6}$ Adoptamos aquí el concepto de matriz de datos que elabora Samaja (1993, p.183) en sentido amplio, como "conjunto estructurado de procedimientos para identificar y hacer referencia a los elementos que integrarán la base empírica de una investigación y para acotar el tipo de predicaciones que se adscribirán a aquéllos".
} 
respuestas obtenidas de las encuestas. De esta forma, determinamos las siguientes categorías:

- Pregunta 1: agrupamos los resultados en "Le gusta leer" o "No le gusta leer". Respecto del tipo de lectura de preferencia reunimos los datos considerando como categorías los distintos ámbitos de producción de los textos que los estudiantes leen: literario, periodístico, académico y religioso.

- Pregunta 2: diferenciamos entre los que contestaron afirmativamente esta pregunta como "Escribe a menudo" y los que señalaron que escriben ocasionalmente como "Escribe poco o nada". Al igual que en la pregunta anterior, categorizamos las respuestas tomando en cuenta los ámbitos en los que los estudiantes escriben: familiar (ej. mensajes de textos, mails), laboral, académico e íntimo (ej. composición de canciones, cuentos, poemas).

- Pregunta 3: sobre las dificultades que los estudiantes expresan, en primer lugar, reunimos las respuestas afirmativas, por un lado, y las negativas, por el otro. En segundo lugar, dividimos las respuestas entre quienes mencionan dificultades en la lectura y quienes señalan problemas en la escritura. Por último, para el análisis de las dificultades en la escritura, adoptamos y adaptamos la clasificación propuesta por Cassany (1998). Categorizamos, así, las dificultades en relación con las normas de la lengua, la coherencia, la cohesión, la adecuación y problemas generales. En la categoría de adecuación, no nos referimos únicamente a la variedad y al registro, sino también incluimos la adecuación genérica, que refiere a los rasgos específicos de los diversos textos que se producen y circulan en la universidad.

- Pregunta 4 (primera encuesta): en esta pregunta, ordenamos los resultados en función de las expectativas que los mismos estudiantes mencionaron: aprobar, aprender, superarse, establecer lazos afectivos, recuperar conocimientos.

- Pregunta 4 (segunda encuesta): distinguimos las respuestas afirmativas de las negativas. Para indicar cuáles fueron los cambios más destacados, nos valimos de la clasificación de Cassany (1998) utilizada para la pregunta 3, a la que le sumamos las modificaciones en la comprensión lectora.

Luego de esta primera etapa, procesamos la información recolectada a través de la cuantificación de los datos que se desprenden de las respuestas obtenidas en las encuestas. 
De esta forma, pudimos establecer las referencias porcentuales y detectamos las tendencias predominantes.

En un segundo momento, siguiendo a Vasilachis (2006), utilizamos una metodología cualitativa interpretativa, con el fin de reflexionar en torno a los porcentajes obtenidos. De acuerdo con esta investigadora, la metodología cualitativa se interesa por la forma en la que el mundo es comprendido, experimentado y producido, por el contexto y por los procesos, por la perspectiva de los participantes, por su experiencia y sus conocimientos. Esta metodología es inductiva, multimetódica y reflexiva.

Por otra parte, además del análisis y de la interpretación de los resultados, hemos incluido algunas respuestas que consideramos son representativas de la muestra con el propósito de precisar y profundizar el alcance del estudio realizado.

\subsection{Características de la población analizada}

La primera encuesta fue realizada a 303 personas (222 mujeres y 81 hombres) entre 17 y 55 años. La segunda se aplicó a 171 alumnos: 96 mujeres y 75 hombres. Las edades de este grupo variaron entre los 18 y los 75 años. Los datos relativos a la escolaridad de los estudiantes indican, en el primer cuestionario, que la mayoría de los encuestados ingresantes a la universidad cursó únicamente el secundario (el 85,9\%). Un 5,9\% tiene estudios terciarios incompletos; un 2,3\%, estudios terciarios completos; y un 5,9\%, estudios universitarios incompletos. Es decir, ninguno de los encuestados finalizó una carrera universitaria.

Es interesante observar las respuestas recabadas en esta misma pregunta en la segunda encuesta: nos revelan que el 69,6\% tiene estudios secundarios completos; el 4,7\%, estudios terciarios incompletos; el 8,2\%, estudios terciarios completos; y el 0,6\%, estudios universitarios completos. Solamente el $16,9 \%$ afirma tener estudios universitarios incompletos. Esta cifra es significativa ya que nos advierte que existe cierto reparo por parte de los estudiantes en apropiarse e instalarse en el lugar de sujeto académico: todos ellos son estudiantes universitarios. Finalmente, en relación con los talleres o cursos previos de lectura y/o escritura cabe destacar que la mayoría no realizó ninguno. En la primera encuesta, solamente el $1,6 \%$ señala haber asistido a alguna actividad relativa a estas prácticas y, en la segunda, el $4,7 \%$. 


\section{Resultados ${ }^{7}$}

\subsection{Las prácticas de lectura y escritura, y la demanda del Otro académico}

Para comenzar con el análisis de las encuestas, en este apartado, nos dedicaremos a las preguntas 1 y 2 que aspiran a reflexionar en torno a las prácticas de lectura y de escritura de los encuestados y que nos permiten empezar a puntear algunas cuestiones sobre las representaciones que circulan en el imaginario de los alumnos. Recordemos estas preguntas: 1. ¿Le gusta leer? Si es así, ¿qué tipo de lecturas prefiere?, 2. ¿Escribe a menudo? ¿En qué momentos? Examinaremos los resultados arrojados por las dos encuestas. (Ver figuras 1 y 2 )

Figura 1. Porcentajes de las respuestas sobre por la lectura

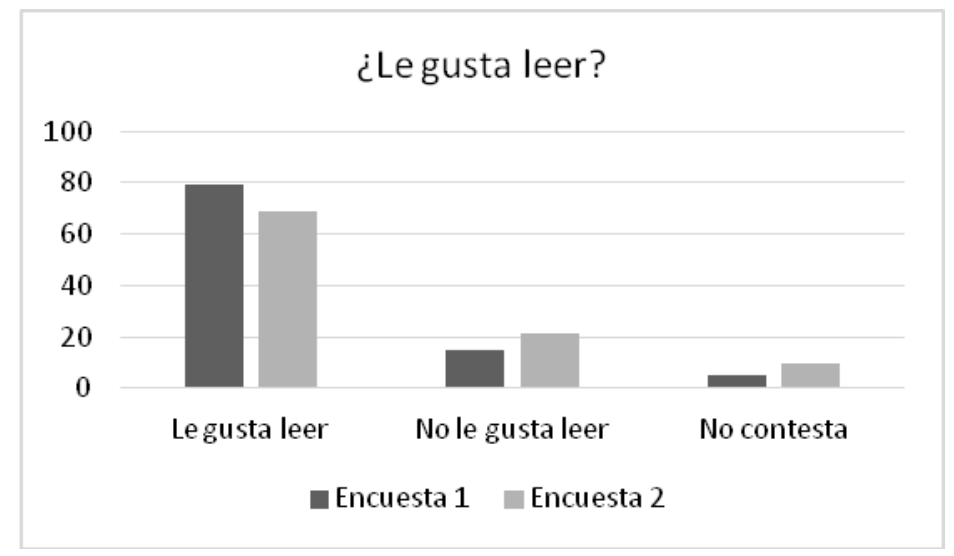

Figura 2. Porcentajes de las respuestas sobre el tipo de lectura.

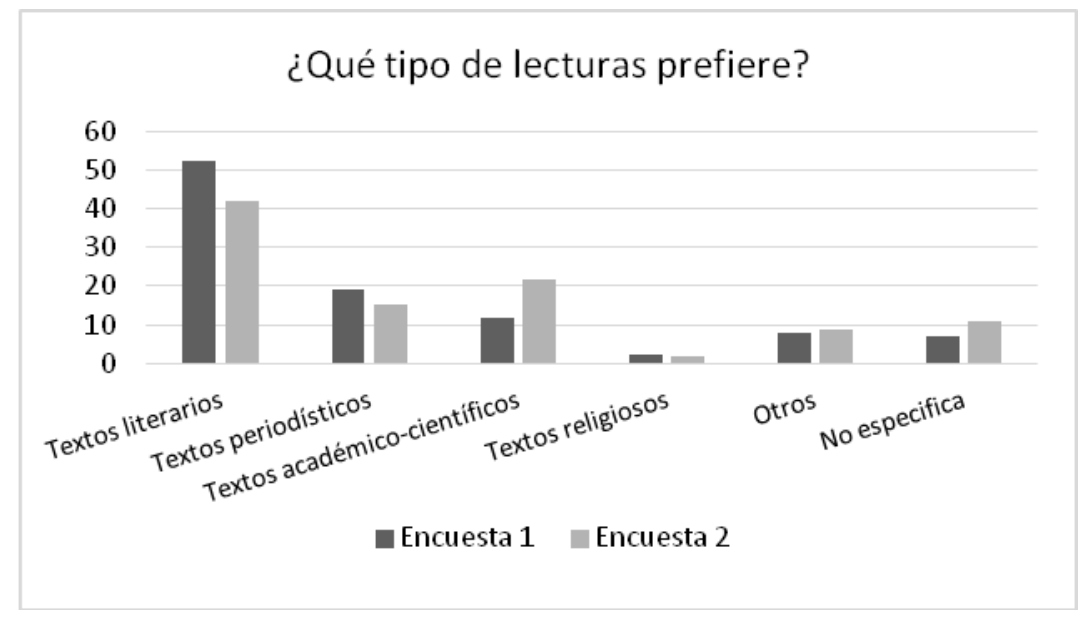

${ }^{7}$ Dado que los resultados de la investigación arrojaron que no existen cambios sustantivos al tomar en cuenta las variables seleccionadas (sexo, escolaridad y experiencia previa en talleres de escritura), se decidió, a los fines expositivos, no incorporar estas variables en el análisis de datos. 
Ante la pregunta sobre la lectura, en la primera encuesta, un 79,5\% de los encuestados afirma que le gusta leer, un $15,2 \%$ manifiesta que no le gusta la lectura y un $5,3 \%$ no contesta. En la segunda encuesta, un $69 \%$ de los estudiantes señala que le gusta leer, un $21,6 \%$ refiere que no le gusta la lectura y un 9,4\% no responde esta pregunta. Estos primeros resultados indican que al término del año académico no hubo una modificación sustancial respecto de cómo se percibe esta práctica y reflejan que una mayoría de alumnos -previo al comienzo de la carrera y con posterioridad a su ingreso- reconoce cierto interés por leer.

Sin embargo, consideramos que el porcentaje de estudiantes que no le gusta la lectura en la segunda encuesta (el 21,6\%) es significativo por dos cuestiones: por un lado, porque es superior al porcentaje que se desprende del primer cuestionario; por el otro, porque los encuestados ya son estudiantes universitarios. Inclusive, muchos de estos alumnos son los que confiesan que leen solamente cuando esta actividad les es solicitada. Veamos algunas respuestas provenientes de este segundo cuestionario:

1. "No me gusta leer, solo cuando es necesario para estudiar". 8

2. "Sinceramente no me gusta mucho la lectura, en todo caso leo solo para estudiar o responder preguntas".

3. "no, generalmente leo para la facultad si piden leer algo para estudiar".

4. "No me gusta mucho leer. Leo por cumplimiento de mis tareas y para desarrollar mi conocimiento".

En estas respuestas nos encontramos que la representación que se refleja sobre la práctica lectora no está asociada a una actividad placentera, orientada por el deseo de saber, ni realizada siguiendo una iniciativa personal.

Sobre el tipo de textos que los estudiantes leen, en la primera encuesta, un número importante de encuestados establece que la literatura es su lectura de preferencia $(52,4 \%)$. Luego, le sigue la lectura de textos periodísticos (19\%), textos relativos a diferentes campos del saber académico-científico (11,6\%) y textos sobre religión (2,2\%). De estas respuestas, se desprende, en esta primera instancia, que la lectura está asociada principalmente a la lectura literaria y recreativa y, luego, a la lectura informativa. En la segunda encuesta, la literatura sigue estando en un primer lugar (41,9\%); después, se registra la lectura de textos provenientes del ámbito académico-científico $(21,7 \%)$, del ámbito periodístico $(15,1 \%)$ y,

\footnotetext{
${ }^{8}$ Las citas son fieles al original, por lo que mantienen, en algunos casos, los problemas de normativa.
} 
finalmente, del ámbito religioso (1,7\%). Estos resultados determinan que solamente 37 estudiantes (recordemos, de un total de 171) aseguran que los textos relativos a sus estudios universitarios son sus textos de preferencia.

Observamos, entonces, en estas encuestas que la lectura recreativa sigue ubicándose en un primer plano y que la práctica de lectura propia de la formación universitaria -que exige otras competencias- no aparece consolidada aún. Por lo tanto, podemos pensar que en la representación dominante sobre la lectura de este grupo de estudiantes subyace la creencia de que "leer es leer literatura".

Figura 3. Porcentajes de las respuestas sobre la escritura

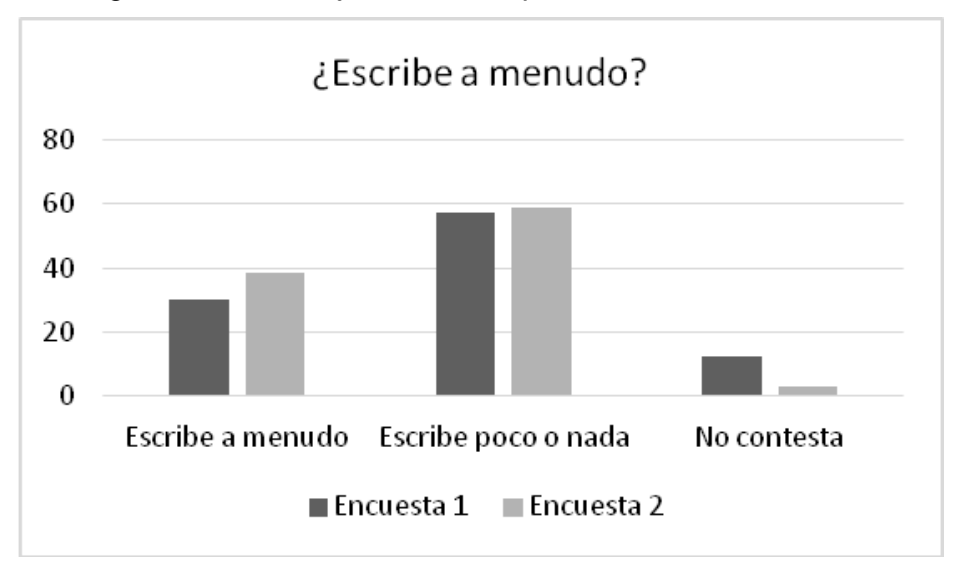

Figura 4. Porcentajes de las respuestas sobre los momentos en los que el alumno escribe.

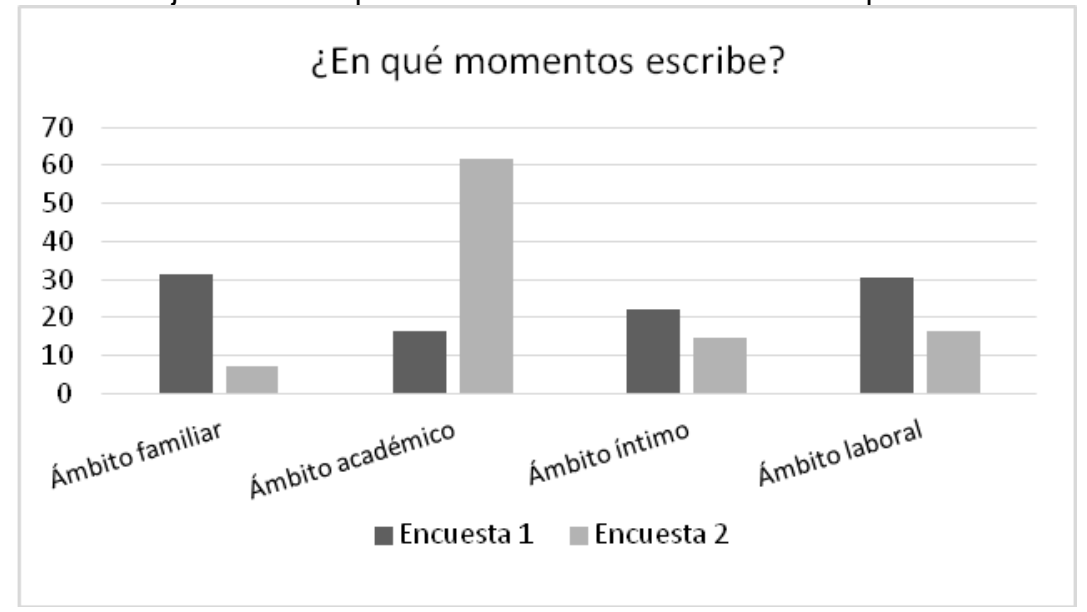

Con respecto a las prácticas de escritura, antes de comenzar a cursar la carrera, un $30,4 \%$ de los estudiantes afirma que escribe frecuentemente; un $57,4 \%$, que escribe poco 0 
nada; y un 12,2\% no contesta (Figura 3). Es curioso observar que, luego de cursar un año en la universidad estos porcentajes varían ligeramente. En efecto, en la segunda encuesta un $38,6 \%$ responde que escribe a menudo, un 58,5\% considera que escribe poco o nada y un $2,9 \%$ no responde. Al igual que en los resultados de la pregunta anterior, notamos que en el caso de la escritura tampoco se registra una práctica afianzada.

En relación con los momentos en que los alumnos escriben (Figura 4), en la primera encuesta, encontramos que, entre los que contestaron esta parte de la pregunta, la mayoría señala que utiliza la escritura en el ámbito familiar (31,2\%) y en el laboral (30,3\%). El ámbito íntimo se registra en tercer lugar $(22,1 \%)$ y, finalmente, el ámbito de estudio aparece en última instancia (16,4\%). Este punto es el que se modifica de manera decisiva en la segunda encuesta. En efecto, allí un gran número de estudiantes $(61,8 \%)$ coincide en manifestar que escribe diferentes géneros académicos a la hora de estudiar (resúmenes, trabajos prácticos, monografías, apuntes). Luego, aparece la escritura en el ámbito laboral $(16,4 \%)$ y como modo de expresión (14,5\%). La escritura como medio de comunicación se desplaza al último lugar (7,3\%). Es importante destacar que, en una gran mayoría de respuestas, al igual que la práctica de lectura, la escritura universitaria se presenta esencialmente frente a la demanda del Otro académico. Veamos algunos ejemplos:

5. "Si, cuando tengo trabajo que presentar en alguna materia".

6. "solo para TPs".

7. "Si, escribo a menudo. Por lo general, cuando tengo que hacer trabajos".

8. "Muy poco, solo cuando me lo piden".

En estos breves fragmentos se advierte que la producción escrita de los alumnos emerge bajo el requerimiento institucional y como instancia de cumplimiento formal. De esta forma, en la representación que los encuestados tienen acerca de la escritura no se manifiesta ni la relación de esta práctica con el estudio ni con su valor formativo.

En suma, a partir de estos primeros resultados, podemos inferir, por un lado, que los estudiantes de primer año todavía no se identifican de manera visible con la imagen de lector/escritor que circula en la universidad: la representación de la lectura como práctica recreativa y, al igual que en la representación de la escritura, como práctica formal, y el escaso número de alumnos que manifiesta escribir frecuentemente así lo demuestra. En este sentido, la lectura y la escritura como respuestas frente a la demanda del Otro académico

\footnotetext{
${ }^{9}$ Aclaración: trabajos prácticos.
} 
borran el deseo de saber propio de la instancia académica y el lugar que ambas ocupan en la formación universitaria y profesional.

\subsection{La lectura en voz alta y la escritura gramatical}

En este apartado nos interesa abordar las respuestas a las preguntas 3 y 4 de la primera encuesta, a saber: 3. ¿Tiene alguna dificultad con la lectura o la escritura? ¿Cuáles? (Ver figuras 5, 6 y 7). 4. ¿Qué expectativas tiene en relación con este curso? Estos resultados nos permitirán reflexionar sobre el reconocimiento o no de dificultades al momento de leer y de escribir, y nos permitirán profundizar sobre las representaciones en torno a la lectura y a la escritura de los ingresantes a la universidad

Figura 5. Porcentajes de las respuestas sobre el reconocimiento de las dificultades

¿Tiene alguna dificultad con la lectura o escritura?

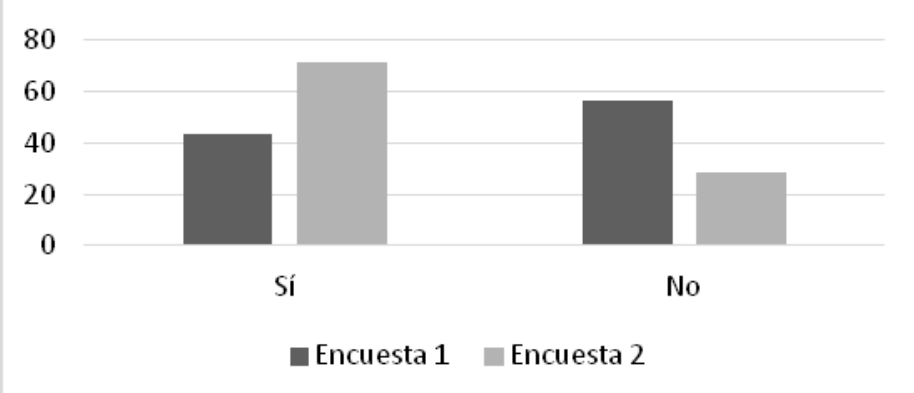

Figura 6. Porcentajes de las respuestas discriminando problemas en la lectura o en la escritura

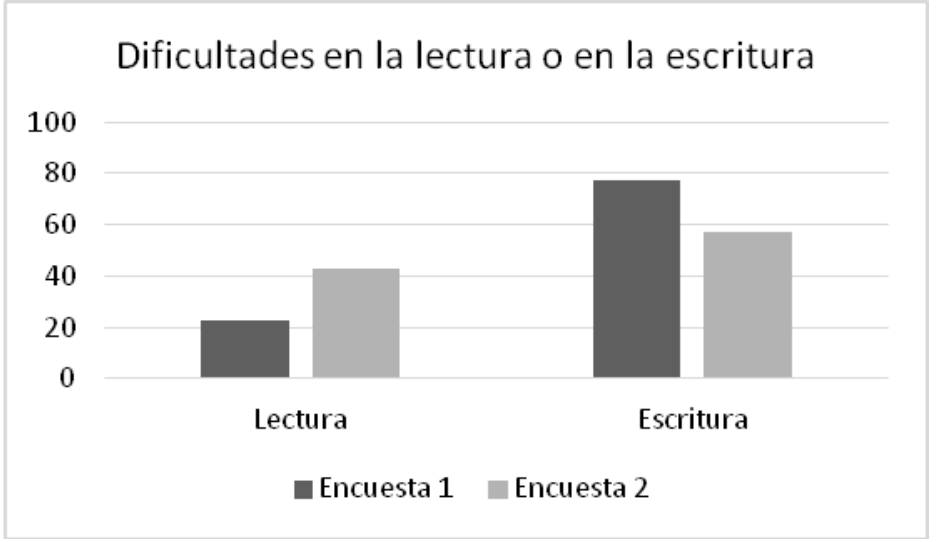


Figura 7. Porcentajes de las respuestas sobre el tipo de dificultad en la escritura

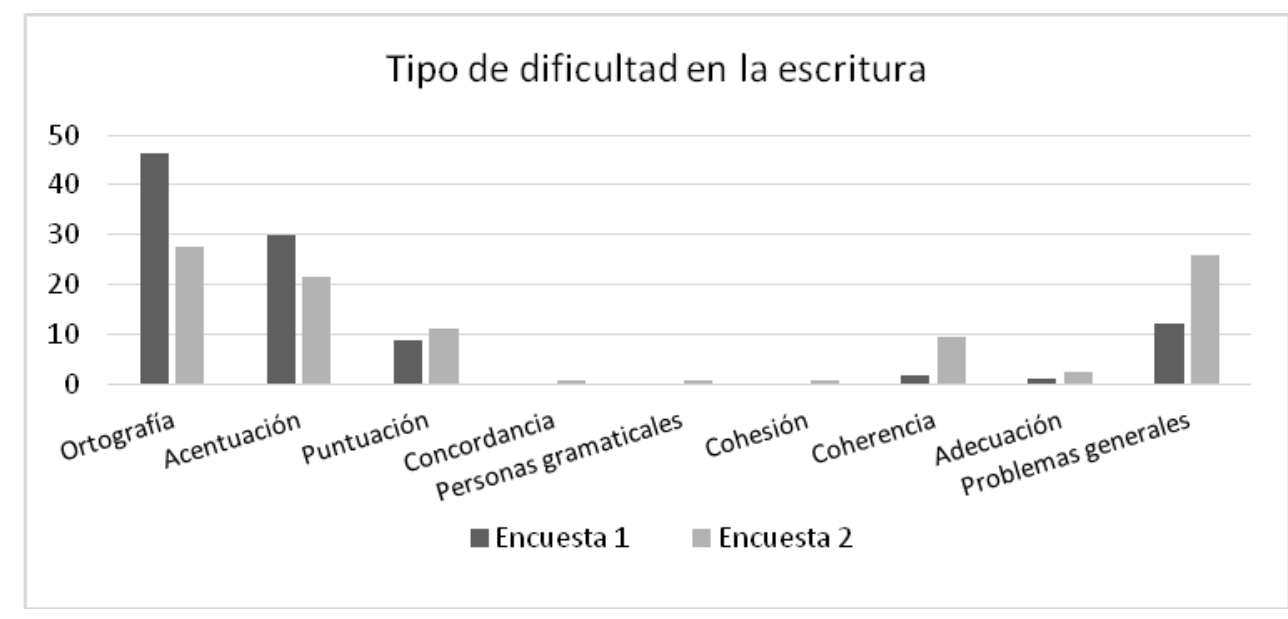

La pregunta por la dificultad en la lectura y/o en la escritura es una pregunta cuya respuesta es particularmente interesante para el desarrollo de un taller, ya que la percepción o no de problemas en alguna de estas prácticas podría llegar a incidir en el interés del alumnado por mejorar o no su competencia en la lecto-escritura. Si tomamos en consideración las respuestas de la totalidad de los ingresantes de la primera encuesta nos encontramos que el $56,4 \%$ de los estudiantes afirma no tener ninguna dificultad. Por el contrario, el 43,6\% reconoce algún tipo de problema en alguna de estas prácticas. De estos valores se observa, entonces, que la mayoría se percibe como lectores/escritores competentes.

La segunda parte de esta pregunta, relativa a identificar el tipo de dificultad, nos revela que la mayoría reconoce que presenta más problemas en la escritura $(77,3 \%)$ que en la lectura $(22,7 \%)$. En esta primera encuesta se marcan dificultades en la normativa: en la ortografía (46,5\%), en primera instancia; en el uso de las tildes $(29,8 \%)$, en segundo lugar; y en el empleo de los signos de puntuación $(8,8 \%)$, en tercer lugar. Dos alumnos $(1,8 \%)$ apuntan problemas en la coherencia, en particular, en la organización textual. Un único alumno $(0,9 \%)$ destaca dificultades en la adecuación, específicamente, en la precisión léxica. Por último, como problemas generales, se destacan aquellos vinculados a la caligrafía $(6,1 \%)$ y a la redacción $(6,1 \%)$.

En función de los resultados obtenidos, notamos que en la representación sobre la escritura emerge su relación con la normativa: "escribir es escribir de acuerdo a las normas de la lengua". Esta visión -restringida y escolar- supone que la realización eficaz de esta práctica está en sintonía con el conocimiento y el manejo gramatical y ortográfico de la 
lengua. Por otra parte, se observa que este conocimiento no es pensado como un conocimiento que ayude a orientar la comprensión y que determina sentidos. Por el contrario, se debe conocer la norma no para mejorar la propia expresión sino para escribir 'bien'. La norma está pensada en términos de corrección y no de sentido, lo que conlleva entender el lenguaje exclusivamente como código. Veamos lo que afirma una alumna que plantea que no tiene dificultades al momento de escribir:

9. "Me gustaría aprender reglas de acentuación ya que considero que una persona no sabe escribir si no cumple estas reglas".

Asimismo, de las respuestas de los estudiantes que refieren problemas en la lectura, se deduce que la representación de esta práctica se asocia, principalmente, con la lectura en voz alta. Un número muy bajo de estudiantes (3\%) manifiesta problemas en la comprensión de textos.

Figura 8. Porcentajes de las respuestas sobre expectativas

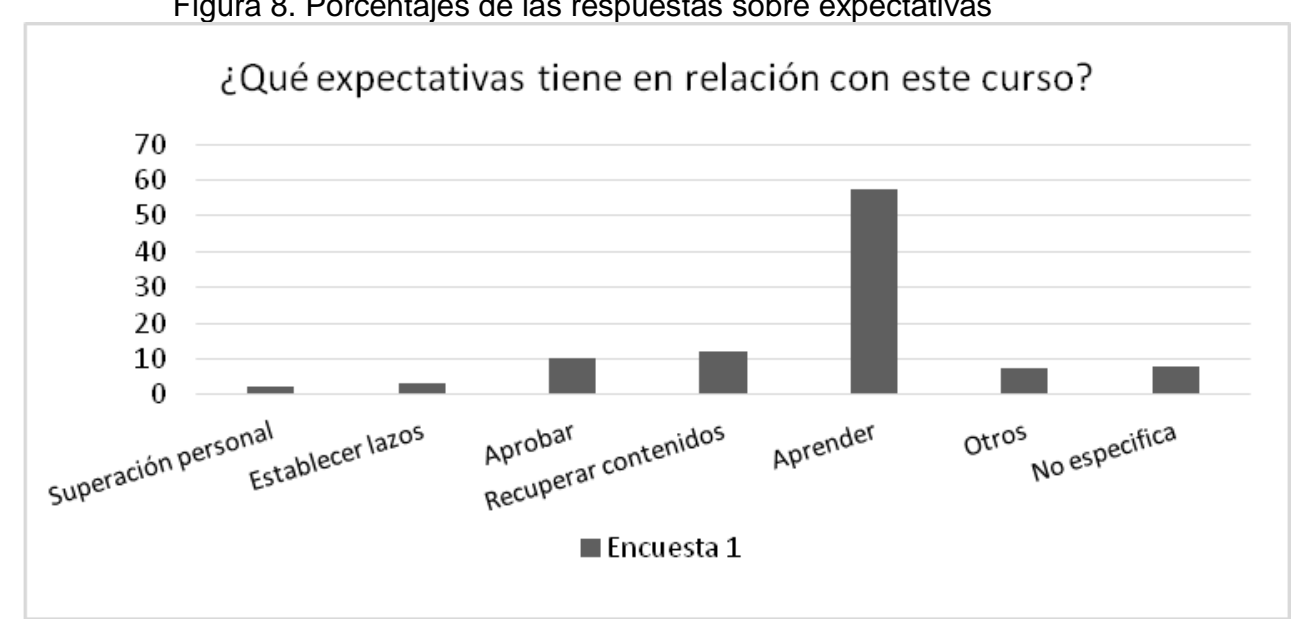

Finalmente, si incorporamos a estos resultados las respuestas de los alumnos sobre las expectativas en torno al curso, advertimos que, a pesar del no reconocimiento de sus propias dificultades (Figura 8), en la mayoría de los encuestados (57,4\%) se destaca el interés de los alumnos por "mejorar" y "aprender". Dentro de las expectativas, encontramos respuestas que van desde la propia superación (2\%) hasta armar lazos y vínculos afectivos con los demás estudiantes (3\%). Un gran número de ingresantes plantea como expectativas la de recuperar los contenidos trabajados en la escuela media (12,2\%) y también la de aprobar (10\%). Pero, en la mayoría de los casos, los estudiantes hacen referencia a aprender y a adquirir nuevos conocimientos: 
10. "Aprender más sobre la lectura y la escritura".

11. "Espero poder aprender más y sobre todo seguir adelante".

12. "Aprender a comprender más fácilmente los textos y a tener una buena escritura".

13. "Que me ayude a expresarme mejor, y a comprender mejor los textos de la universidad".

14. "Aprender y comprender los temas. Mejorar la escritura, perfeccionar".

De estas respuestas, sobresale, entonces, el interés de los estudiantes por mejorar la comprensión lectora y la escritura. De allí que se pueda afirmar que tanto en la representación de la lectura como de la escritura de los ingresantes ambas prácticas se presentan como prácticas pasibles de ser transformadas. En este sentido, la lectura y la escritura no son concebidas solamente como 'productos' de una actividad, sino también como 'procesos'.

\subsection{La lectura y la escritura, y la heterogeneidad}

¿Qué sucede luego de cursar el Curso de Preparación Universitaria de Lengua, el Taller de Lectura y Escritura y otras materias de la universidad? ¿Se produce alguna modificación en las representaciones del alumnado sobre sus propias dificultades y sobre el modo en que conciben ambas prácticas? Intentaremos responder estas interrogantes analizando las últimas dos preguntas de la segunda encuesta: 3. ¿Tiene alguna dificultad con la lectura o la escritura? ¿Cuáles?, 4. ¿Cuál es su opinión sobre el Taller de Lectura y Escritura? ¿Hubo cambios en su escritura o en la lectura a partir del Taller? ¿Cuáles?

En relación con la pregunta sobre las dificultades, nos encontramos con que los porcentajes de la primera encuesta -que indicaban que la mayoría no las percibía- se encuentran significativamente modificados en el segundo cuestionario (véase la figura 5). En este sentido, mientras que el $28,6 \%$ de la población encuestada no reconoce problemas cuando lee o escribe, el 71,4\% advierte que sí las tiene. Estos resultados nos permiten señalar que la mayoría de los estudiantes que ingresa a la universidad no repara en sus dificultades frente a la lectura y/o escritura de textos y es en ese encuentro -entre el sujeto universitario y la práctica concreta del estudiante- en donde comienzan a interrogar el saberleer y el saber-escribir propios.

Respecto del tipo de problema, estas encuestas revelan que el porcentaje de alumnos que encuentra dificultades en la práctica de lectura se elevó notablemente: un 42,6\% de los 
encuestados reconoce dificultades en la lectura, específicamente, en la comprensión lectora. Un 57,4\% afirma, por otra parte, que tiene problemas en la escritura (Véase figura 6). A propósito de la escritura, se mencionan problemas en el uso de la normativa: ortografía $(27,4 \%)$, acentuación $(21,4 \%)$, puntuación $(11,1 \%)$, concordancia $(0,8 \%)$, uso de personas gramaticales $(0,8 \%)$. También, se señalan problemas vinculados con la coherencia $(9,4 \%)-$ organización y desarrollo textual-, la cohesión $(0,8 \%)$-repetición de palabras-, la adecuación -la precisión léxica $(0,8 \%)$, la argumentación $(0,8 \%)$, la reformulación $(0,8 \%)$ Finalmente, problemas generales como caligrafía $(3,6 \%)$ y redacción $(22,3 \%)$ (véase figura 7).

Estos resultados nos permiten observar la aparición de nuevas categorías que antes no figuraban: algunas de ellas relativas a la normativa y otras no. Esto nos indica, por un lado, una mayor diversidad en las respuestas y, por el otro, una representación más compleja de lo que implica y conlleva la práctica de escritura. Ahora bien, si tomamos el conjunto de los resultados nos encontramos que más del $60 \%$ enumeró dificultades asociadas a la normativa. Este dato indicaría, en principio, que la representación de la escritura entendida en términos de código sigue ocupando un lugar privilegiado en el imaginario de los estudiantes.

Figura 9. Porcentajes de las respuestas sobre los cambios en las prácticas de lectura y/o escritura

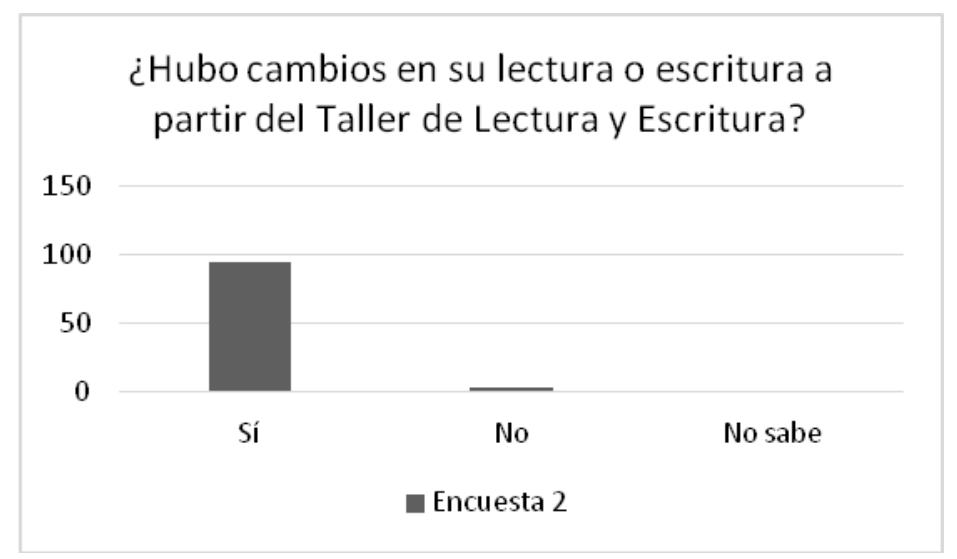




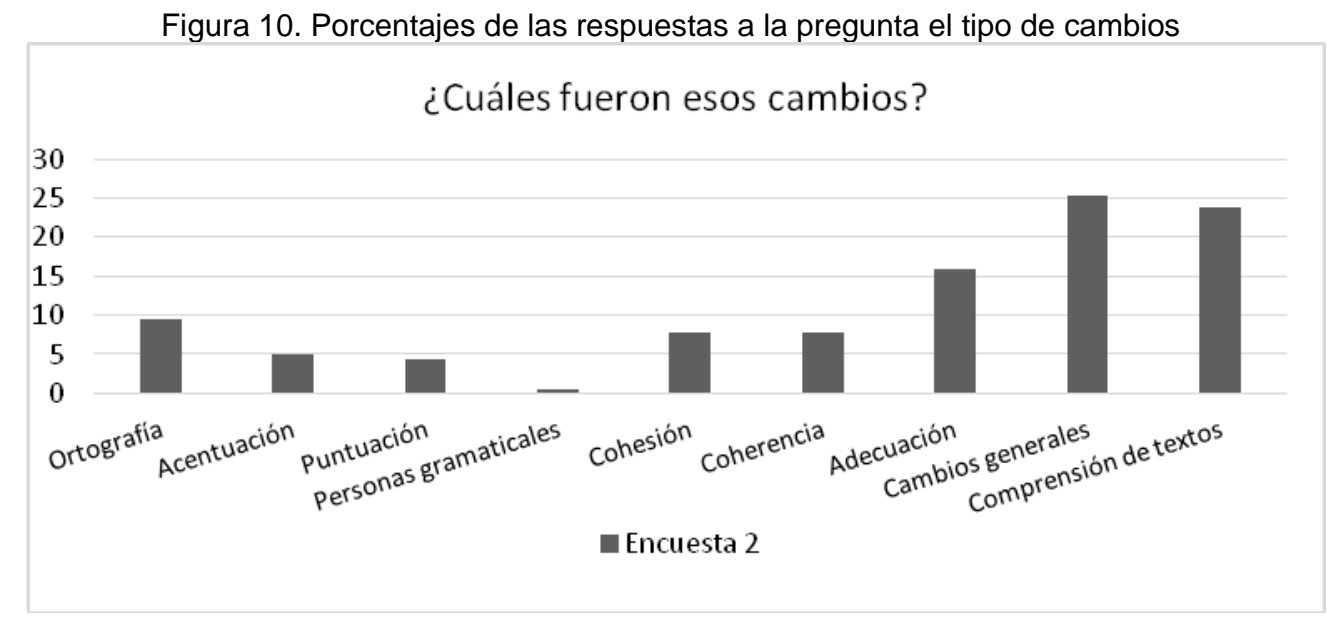

En la pregunta por los cambios producidos a partir de la cursada del Taller, encontramos, al igual que en la pregunta anterior, una diversidad de respuestas (Figura 9). De la totalidad de encuestas, solamente el 3,5\% considera que no hubo modificaciones en sus modos de leer y/o escribir y el 1,2\% no está seguros si los hubo. Una amplia mayoría (95,3\%) percibe que hubo modificaciones luego de cursar esta asignatura. Sobre qué cambios se produjeron, un porcentaje alto de estudiantes (27\%) no puede especificar cuáles fueron. Los restantes mencionan cambios relativos a normativa (Figura 10): puntuación $(4,4 \%)$, ortografía $(9,4 \%)$, acentuación $(5 \%)$, empleo de personas gramaticales $(0,6 \%)$. Se destacan, asimismo, aquellos vinculados a la cohesión $(7,7 \%)$-conectores- y a la coherencia $(7,7 \%)$-organización y desarrollo textual-. También, se registran modificaciones relacionadas con la adecuación: armado de bibliografía (0,6\%), elaboración de géneros universitarios (informe y resumen) y tipos textuales (13,2\%), precisión léxica (2,2\%).

Los cambios generales que se nombran son: la redacción $(20,4 \%)$, el prestar más atención al escribir (2,8\%), los cambios en el modo de razonar y de confrontar autores $(1,6 \%)$ y la reescritura (0,6\%). Observamos en estos valores una distribución más pareja de los porcentajes y la aparición de categorías que no figuran en la respuesta anterior. En esta pregunta, nos encontramos que en la representación de la escritura esta práctica no solamente se presenta asociada a la normativa sino a una multiplicidad de variables que convergen en ella. Escribir no es simplemente "escribir de acuerdo con las normas de la lengua", sino también, entre otros aspectos, "escribir coherente y cohesivamente, adecuando el texto al género y al tipo textual; implica reescribir y corregir, poder distinguir diferentes voces, y relacionar y confrontar autores". 
Teniendo en cuenta estos resultados podemos destacar dos puntos primordiales a propósito del imaginario que circula sobre la lectura y la escritura: la aparición de un lector/escritor universitario en formación y la tensión que emerge en las representaciones sobre la escritura. En primer lugar, la pluralidad en las respuestas y la distribución más equitativa de los porcentajes nos revelan que los estudiantes, luego de cursar el Taller, comienzan no solo a construir una mirada más compleja en torno a lo que implica la escritura sino también a encontrarse con sus propias prácticas y, por ende, con un sujeto que se interroga sobre su quehacer. Inclusive, la problemática de la comprensión textual, prácticamente ausente en la primera encuesta, emerge aquí con mayor fortaleza. El alto porcentaje de estudiantes que no puede especificar qué cambios se realizaron luego de cursar el Taller y de aquellos que no pueden identificar claramente sus dificultades nos refuerza, sin embargo, el carácter de formación que acompaña la noción de lector/escritor universitario y la exigencia que estas actividades demandan.

En segundo lugar, estos datos nos remiten a que la dificultad se sigue pensando en términos de normativa. En este sentido, pareciera que las nuevas variables discursivas que se despliegan y se incorporan luego de cursar el Taller se evidencian como a-problemáticas. Veamos un ejemplo. Un estudiante responde de la siguiente manera las preguntas 3 y 4 :

15. "Si. Los tildes y las faltas de ortografia."

16. "De gran ayuda para incorporar una mejor y coherente redacción de textos, informes, monografías, etc."

En estas respuestas, observamos que aquello que se reconoce como dificultad es el uso correcto de normativa, mientras que la elaboración de un género académico, que involucra aspectos de diversa índole, como un informe o una monografía, no pareciese despertar ningún conflicto.

Otra alumna contesta lo siguiente en la pregunta 4:

17. "Es un taller clave para el desarrollo de la carrera. Si, los cambios se produjeron en la escritura. Por ejemplo, en el último parcial tuvo 4 errores de tildación, antes escribia peor."

Vemos el lugar medular que ocupa en este caso el uso correcto de los acentos: la estudiante llega, incluso, a contabilizarlos. 
¿Cómo podrían explicarse, entonces, estos resultados? En relación con esto, creemos, por un lado, que estas respuestas reflejan cierta tensión entre las representaciones con las que ingresan los estudiantes y los cambios que se producen a partir de Taller. Como señalamos anteriormente, las representaciones presentan un carácter dinámico: es, entre otros espacios, en el marco de la enseñanza donde representaciones previas se confrontan con nuevos saberes y nuevas prácticas, dando lugar a procesos de apropiación paulatinos y graduales. Por el otro, pensamos que concebir la dificultad en la escritura en relación con la normativa tranquiliza, ya que la normatividad explícita introduce la posibilidad de control.

En efecto, la codificación del lenguaje reduce la imprecisión, la apertura -clausura, por el contrario-, la incertidumbre del papel en blanco, el abismo que implica pensarse como sujetos lectores/escritores, con un abanico de posibilidades (Bourdieu, 1987). La sistematización permite una formalización concreta. En esta representación, conocer las reglas implica conocer el lenguaje. Bourdieu (1987, p.84) afirma que se puede enunciar como ley general que "cuanto más peligrosa es la situación, más la práctica tiende a ser codificada". La codificación es una puesta en orden simbólica, establece fronteras tajantes, en tanto opera cortes netos.

Es por esta razón por la cual lo que puede ser controlado es lo que puede percibirse como dificultad. Pensar, por otra parte, la escritura como un conjunto de variables que confluyen entre sí, variables relativas a la enunciación y al discurso, introduce una grieta por donde el sujeto puede manifestarse y, por ende, "equivocarse". Estas variables también presentan un grado de sistematización pero su codificación no es tan rigurosa ni precisa. En este sentido, involucran un margen mayor de subjetividad y no responden a un control inmediato: reconocer y reconocerse en estas dimensiones reclama un mayor nivel de exigencia. Demanda un conocimiento más profundo del sujeto como lector/escritor.

\section{Palabras finales}

Al comienzo de este artículo nos hemos referido a los manuales y a los cursos, materias y/o talleres sobre lectura y escritura universitarios. Los manuales que se proponen como objetivo acercar a los estudiantes a los modos de decir y de leer académicos conciben a un alumno que no cuenta con esta formación lecto-escritora, propia de las instituciones superiores. De allí que sea necesario -según estos textos- que el alumno conozca y se entrene en las convenciones discursivas de la comunidad académica. En tal sentido, el supuesto implícito, como ya hemos afirmado, es que la lectura y la escritura académicas 
participan de la construcción del sujeto universitario. Este mismo supuesto subyace en las materias y/o talleres de lectura y de escritura que se dictan en el ingreso y/o en las carreras de grado de diversas universidades argentinas y que, en la actualidad, tienen una amplia difusión. Estas materias que apuntan a un nivel metauniversitario, también aspiran a alcanzar este ideal. En ambos casos, se parte de un sujeto vacío empíricamente. No se conoce nada de él; solo se sabe qué se espera de su desempeño: un saber-leer y un saber-escribir universitarios.

El sujeto universitario es, por lo tanto, un sujeto sujetado por este Otro institucional que, en este mismo movimiento, se presenta en su carácter normalizador y disciplinador. No solamente disciplina desde los saberes que se despliegan, sino también desde las formas discursivas que allí circulan. Impone aquello que se dice, pero también, cómo se lo dice. De esta forma, instala y regula sus verdades dentro de un eje discursivo de correcciónincorrección que remite a un sistema de normas y censuras específicas ${ }^{10}$.

Frente a esta sujeción, el estudiante responde con su propio imaginario, el cual se pone en juego en las prácticas concretas. Como ya lo hemos afirmado, las representaciones sobre la lectura y la escritura operan sobre los modos en que los sujetos leen y escriben, y en cómo se posicionan como lectores/escritores universitarios. En este sentido, participan de la construcción de la identidad social y evidencian la relación de los sujetos con la comunidad académica.

Luego del análisis de los resultados de las encuestas, esta investigación ha detectado, por un lado, modificaciones en las representaciones sociales en torno a la lectura y a la escritura de los estudiantes de la UNAJ al término del primer año lectivo y, por el otro, ciertas continuidades que ponen de relieve la tensión que surge del conflicto entre las representaciones previas de los alumnos y los nuevos saberes que se demandan.

Se ha concluido, en primer lugar, que al momento del ingreso, las representaciones dominantes sobre la lectura y la escritura de los estudiantes se distancian de aquellas que están latentes en las prácticas de lectura y escritura exigidas por la institución. En efecto, estas representaciones se nutren, en esta instancia, de una visión restringida y escolar, que borra la complejidad de los procesos y las numerosas variables que deben tomarse en cuenta para alcanzar una lectura y una escritura académicamente 'exitosas'. Los

\footnotetext{
${ }^{10}$ Como no era un propósito de este artículo, no hemos abordado aquí la tensión existente entre las exigencias institucionales y la especificidad de los distintos campos del conocimiento. Hemos analizado esta problemática en trabajos anteriores (véase Savio, 2009a, 2009b, 2010a, 2010b).
} 
encuestados asocian la lectura con la literatura, principalmente, y la acción de leer con la 'lectura en voz alta'.

La representación de la escritura, por su parte, está vinculada con la corrección normativa exclusivamente, eliminando las dimensiones enunciativas, discursivas y textuales que se entrecruzan en todo escrito. Sin embargo, la expectativa de que se puede aprender y mejorar introduce intuitivamente la noción de proceso que caracteriza ambas prácticas. Por otra parte, este imaginario, en el que se reduce la complejidad de la lectura y de la escritura, está en sintonía con el escaso reconocimiento de las propias dificultades de los alumnos al momento de leer o de escribir. En este punto, se podría pensar que esta falta de conciencia respecto de sus competencias como lectores/escritores incide negativamente al pensar la autocorrección, una de las etapas primordiales a la hora de escribir un texto y de trabajar a partir de la lectura de un escrito universitario.

En segundo lugar, se ha detectado que, al finalizar el año lectivo y al término de la cursada del Taller de Lectura y Escritura, la identidad universitaria es aún incipiente, ya que la imagen de lector/escritor universitarios comienza a aparecer tímidamente: es un lector/escritor en formación. La incorporación del estudiante a la comunidad académica responde, pues, a un proceso gradual y paulatino. En efecto, en este segundo momento, las prácticas de lectura y escritura no se presentan como prácticas académicas consolidadas. La lectura y la escritura aparecen como respuestas frente al pedido del Otro, por lo que no se advierte su valor de formación y el deseo de saber asociado a ellas.

En las representaciones sobre la lectura y la escritura, la literatura y la normativa siguen ocupando un lugar privilegiado. Incluso, la escritura se percibe como una actividad ocasional. No obstante, de los resultados de las encuestas se visualizan varios cambios en las representaciones de los encuestados. Por un lado, en la representación de la lectura, esta práctica, aunque sigue estando asociada fuertemente con el ámbito literario, aparece problematizada. En su encuentro con la teoría, los alumnos detectan problemas en la comprensión lectora, lo que revela la complejidad que conlleva una lectura atenta y analítica de los textos universitarios. Por el otro, reconocen mayores dificultades en la escritura, aunque la representación de las dificultades asociada a la normativa aún persiste dentro del imaginario académico.

Asimismo, dan cuenta de diversas modificaciones producidas en sus propias prácticas luego de haber cursado el Taller, que no remiten únicamente al plano gramatical, lo que implica no solo la elaboración de una representación más compleja sobre el lenguaje sino 
también el surgimiento embrionario de sus propias voces como escritores académicos. De lo homogéneo surge, entonces, lo heterogéneo.

Para finalizar, queremos señalar que es esencial que el docente recupere las representaciones que se encuentran latentes en el quehacer académico, que con el fin último de problematizarlas y desnaturalizarlas, operar sobre su devenir, y así facilitar la incorporación del ingresante a la comunidad universitaria. Si tomamos aquello que recupera Chartier (1992) sobre el sentido antiguo del término representación, podemos pensar que la representación marca una ausencia y una presencia. En tanto ausencia, algo se pierde, algo resta. Aquello que se representa no está. En tanto presencia, la representación cristaliza, coagula el sentido, ligado a las prácticas. Esta cristalización puede obturar el desempeño del estudiante que ingresa por primera vez a una universidad, cuya identidad académica aún es incipiente.

\section{Referencias bibliográficas}

Arnoux, Elvira y Maite Alvarado. (1997). La escritura en la lectura. Apuntes y subrayado como huellas de representaciones de textos. En María Cristina Martínez (comp.), Los procesos de la Lectura y la Escritura (pp.57-77). Cali: Universidad del Valle.

Arnoux, Elvira y Maite Alvarado. (1998). El registro de las modalidades en los apuntes de un texto fuente argumentativo. Signo y Seña, 8, 287-316.

Arnoux, Elvira y Maite Alvarado. (1999). El apunte: restricciones genéricas y operaciones de reformulación. En María Cristina Martínez (comp.), Comprensión y producción de textos académicos expositivos y argumentativos (pp. 39-63). Cali: Cátedra UNESCO para la lectura y la escritura en América Latina y Universidad del Valle.

Arnoux, Elvira y Roberto Bein (comps). (1999). Prácticas y representaciones del lenguaje. Buenos Aires: EUDEBA.

Arnoux, Elvira, Nogueira, Sylvia y Silvestri, Adriana. (2001). La escritura producida a partir de la lectura de textos polifónicos. Evaluación del desempeño de grupos con diferente entrenamiento lector previo. En María Cristina Martínez (comp.), Aprendizaje de la argumentación razonada (pp. 49-77). Cali: Cátedra UNESCO y Universidad de Cali.

Arnoux, Elvira, Sylvia Nogueira y Silvestri, Adriana. (2002). La construcción de representaciones enunciativas: el reconocimiento de voces en la comprensión de textos polifónicos. Signos, 35(51-52), 129-148.

Arnoux, Elvira, Nogueira, Sylvia y Silvestri, Adriana. (2004). Integración semántica de textos expositivos y reformulación en estudiantes con distinto entrenamiento escolar. Lenguas Modernas, 28-29, 111-130. 
Arnoux, Elvira, Nogueira, Sylvia y Silvestri, Adriana. (2006). Comprensión macroestructual y reformulación resuntiva de textos teóricos en estudiantes de institutos de formación de docentes primarios. Signos, 39(60), 9-30.

Arnoux, Elvira, Nogueira, Sylvia y Silvestri, Adriana. (2007). Habilidades metacomprensivas en estudiantes de profesorado: la formulación de preguntas. Folios, (25), 81-96.

Arnoux, Elvira (dir.) (2009). Pasajes. Escuela media-enseñanza superior. Propuestas en torno a la lectura y la escritura. Buenos Aires: Editorial Biblos.

Bidiña, Ana y Amelia Zerillo. (2013). La lectura y la escritura en el ingreso a la universidad. Experiencias con alumnos y docentes de la UNLaM. Buenos Aires: Universidad Nacional de La Matanza.

Bourdieu, Pierre. (1985). ¿Qué significa hablar? Economía de los intercambios lingüísticos. Madrid: Ediciones Akal.

Bourdieu, Pierre. (1987). Cosas dichas. México D.F.: Editorial Gedisa.

Carlino, Paula. (2003a). Leer textos científicos y académicos en la educación superior: Obstáculos y bienvenidas a una cultura nueva. Uni-Pluri/Versidad, 3(2), 17-23.

Carlino, Paula. (2003b). Leer textos complejos al comienzo de la educación superior: tres situaciones didácticas para afrontar el dilema. Textos. Didáctica de la lengua y la literatura, 33, 43-51.

Carlino, Paula. (2003c). Reescribir el examen: transformando el «epitafio» en una llamada al pie de página. Cultura y Educación, 15(1), 81-96.

Carlino, Paula. (2006). Escribir, leer y aprender en la universidad. Buenos Aires: Fondo de Cultura Económica.

Cassany, Daniel. (1998). 2003. Describir el escribir. Cómo se aprende a escribir. Barcelona: Paidós.

Castorina, José, Barreiro, Alicia, Toscano, Ana Gracia. (2005). Dos versiones del sentido común: las teorías implícitas y las representaciones sociales. En José Castorina (coord.), Construcción conceptual y representaciones sociales. El conocimiento de la sociedad (pp.205-238). Buenos Aires: Miño y Dávila.

Ceresani, Viviana, Garbarini, Mónica, Peirano, Gabriela y Toribio, Daniel. (octubre, 2013). El ingreso en la UNAJ: una política orientada a la inclusión educativa. Ponencia presentada en Primeras Jornadas de Educación y Política, Universidad Nacional de Avellaneda, Buenos Aires, Argentina.

Chartier, Roger. (1992). El mundo como representación. Barcelona: Editorial Gedisa.

Di Stéfano, Mariana y Cecilia Pereira. (1998). Representaciones sociales en el proceso de lectura. Signo y Seña, 8, 317-340.

Hyland, Ken. (2009). Academic Discourse. London: Continuum. 
Ivanič, Roz. (1998). Writing and Identity. The discoursal construction of identity in academic writing. Amsterdam/Philadelphia: John Benjamin Publishing Company.

Jodelet, Denise. (1989). Représentations sociales: un domaine en expansión. En AA.VV., Les représentations sociales (pp. 31-61). París: P. U. F.

Magnanego, Florencia y Savio, Karina. (2006). Sobre las prácticas de lectura y escritura en un taller de la Universidad. En AA.VV., Actas de las II Jornadas de Lectura y Escritura, Universidad Nacional de Catamarca, Cátedra UNESCO, Formato CD.

Moscovici, Serge. (1989). Des représentations collectives aux représentations sociales. En: AA. VV., Les représentations sociales (pp.62-86). París: P. U. F.

Moscovici, Serge e Marková, Ivana. (2003). La presentación de las representaciones sociales: diálogo con Serge Moscovici. En José Castorina (comp.), Representaciones sociales. Problemas teóricos y conocimientos infantiles (pp. 111-152). Barcelona: Gedisa.

Pereira, Cecilia. (septiembre, 2006). La lectura y la escritura en el CBC: memoria de la experiencia en la cátedra de Semiología. Ponencia presentada en el Primer Congreso Nacional: "Leer, escribir y hablar hoy", Tandil, Buenos Aires, Argentina.

Raiter, Alejandro. (2002). Representaciones sociales. En Alejandro Raiter et al., Representaciones sociales (pp. 9-29). Buenos Aires: Eudeba.

Samaja, Juan. (1993). Epistemología y Metodología. Elementos para una teoría de la investigación científica. Buenos Aires: Eudeba.

Savio, Karina. (2009a). Sobre la escenografía en defensas de tesis de psicoanálisis: entre la tradición y la creación. RASAL, 1/2, 77-93.

Savio, Karina. (2009b). El psicoanálisis y los géneros académicos de postgrado (o del retorno de una interminable polémica. Revista Universitaria de Psicoanálisis, 9, 259274.

Savio, Karina. (2010a). Las huellas del autor en el discurso académico: Un estudio de tesis de psicoanalistas argentinos. Lenguaje, 38(2), pp.563-590.

Savio, Karina. (2010b). En torno a la escritura de casos clínicos en el discurso académico. Revista Universitaria de Psicoanálisis, (10), 257-278.

Swales, John. (1990). Genre Analysis: English in academic and research settings. Cambridge: Cambridge University Press.

Vasilachis, Irene (coord.). (2006). Estrategias de investigación cualitativa. Barcelona: Gedisa. 